\title{
Avaliação antropométrica e sua associação com variáveis clínicas em pacientes pediátricos com fibrose cística de um centro no Nordeste brasileiro
}

\author{
Anthropometric assessment and its association with clinical variables in pediatric \\ patients with cystic fibrosis from a center in the northeast of Brazil
}

\begin{abstract}
Jamile Cardoso Bomfim ${ }^{1 *}$, Valmir Machado de Melo Filho², Adson Santana de Jesus ${ }^{3}$, Fernanda Gomes Coqueiro ${ }^{4}$, Edna Lúcia Souza ${ }^{5}$

${ }^{1}$ Nutricionista pela Universidade Federal da Bahia (UFBA). Especialização em Nutrição Clínica. ${ }^{2}$ Estudante de medicina da Faculdade de Medicina da Bahia, UFBA; ${ }^{3}$ Mestrando do Programa de Pós-Graduação em Processos Interativos dos Órgãos e Sistemas, Instituto de Ciências da Saúde, UFBA.; ${ }^{4}$ Nutricionista assistencial no Hospital Universitário Professor Edgar Santos - Universidade Federal da Bahia- UFBA.; ${ }^{5}$ Doutora em Medicina e Saúde. Professora Associada IV do Departamento de Pediatria da Faculdade de Medicina da Bahia, UFBA.
\end{abstract}

\begin{abstract}
Resumo
Introdução: a avaliação e o acompanhamento nutricional fazem parte do cuidado integral dos pacientes com fibrose cística (FC), possibilitando intervenção precoce e tratamento mais efetivo da doença. Objetivo: avaliar o estado antropométrico de crianças e adolescentes com FC e pesquisar sua associação com variáveis clínicas e demográficas. Metodologia: estudo descritivo, incluindo-se indivíduos entre 1-19 anos de idade. Foram registradas medidas de peso, estatura, circunferência do braço (CB) e da prega cutânea tricipital (PCT) e calculados indicadores antropométricos (Peso/Idade- P/I, Altura idade -A/I e IMC/idade-IMC/I) e Circunferência Muscular do Braço (CMB). Comparou-se o indicador CMB com variáveis clínicas e demográficas através do cálculo de razões de prevalência (RP). Resultados: foram incluídos 41 pacientes, 53,6\% do sexo masculino, mediana de idade de 78 meses. Quinze (36,6\%) pacientes foram classificados como desnutridos pela avaliação da $\mathrm{CMB}(<\mathrm{P5})$, enquanto, através do indicador IMC/I, apenas um $(2,4 \%)$ apresentava magreza. Trinta (73,1\%) pacientes tinha insuficiência pancreática (IP), determinada pela terapia de reposição enzimática (TRE) e/ou níveis da elastase fecal. Todos os pacientes realizaram pesquisas de mutações no gene CFTR e 38 (92,8\%) tiveram duas variantes patológicas identificadas, dos quais $25(65,8 \%)$ tinham pelo menos um alelo para a variante F508del. Dezesseis (39\%) crianças cursavam com infecções respiratórias recorrentes. A desnutrição (CMB $<P 5)$ teve uma associação positiva com as seguintes variáveis: idade do diagnóstico, sexo masculino, PCT >P15, TRE, mutação F508/del e A/K-2, observando-se maior associação com as três últimas, RP de 6,25, 3,12; e 2,06; respectivamente. Conclusão: o IMC pode não ser suficiente para a avaliação do estado nutricional na FC, pois subestima a prevalência de desnutrição. Com exceção das infecções respiratórias recorrentes, as demais variáveis tiveram associação com o indicador $\mathrm{CMB}<\mathrm{P} 5$.
\end{abstract}

Palavras chaves: Composição corporal. Mucoviscidose. Antropometria.

\begin{abstract}
Introduction: the assessment and nutritional follow-up are part of the comprehensive care of cystic fibrosis (CF) patients enabling an early intervention and a more effective disease treatment. Objective: to assess the anthropometric status of children and adolescents with cystic fibrosis (CF) and to investigate its association with clinical and demographic variables. Methodology: descriptive study, including individuals between 1-19 years of age. Weight, height, arm circumference (AC) and tricipital skinfold (TSF) measurements were recorded and anthropometric indicators were calculated (Weight/Age-W/A, Height age $-\mathrm{H} / \mathrm{A}$ and BMI/age-BMI/A) and Muscle Circumference of the Arm (AMC). The AMC indicator was compared with clinical and demographic variables by calculating prevalence ratios (PR). Results: 41 patients were included, 53.6\% male, median age 78 months. Fifteen (36.6\%) patients were classified as malnourished by the AMC assessment (<P5), while, through the BMI/A indicator, only one (2,4\%) was thin. Thirty $(73.1 \%)$ patients had pancreatic insufficiency (PI), determined by enzyme replacement therapy (ERT) and/or fecal elastase levels. All patients underwent research for mutations in the CFTR gene and 38 (92.8\%) had two pathological variants identified, of which 25 (65.8\%) had at least one allele for the F508del mutation. Sixteen (39\%) children had recurrent respiratory infections. Malnutrition (AMC $\angle P 5)$ had a positive association with the following variables: age of diagnosis, male gender, TSF > P15, TRE, F508del variant and H/A <-2, with a greater association with the last three, PR of 6.25, 3.12; and 2.06; respectively. Conclusions: BMI may not be sufficient to assess nutritional status in CF since it underestimates the prevalence of malnutrition. Except for recurrent respiratory infections, the other variables were associated with the AMC indicator $<P 5$.
\end{abstract}

Keywords: Body composition. Mucoviscidosis. Anthropometry.

Correspondente/Corresponding: *Jamile Cardoso Bomfim - End: Rua Emídio dos Santo, no 388, Casa 5, Barbalho- Salvador, Ba. - Tel: (71) 9 9174-5031. - E-mail: milebomfim@hotmail.com. 


\section{INTRODUÇÃO}

A avaliação e o acompanhamento nutricional fazem parte do cuidado integral dos pacientes com fibrose cística $(\mathrm{FC})$, sendo essencial para o tratamento efetivo da doença ${ }^{1}$ A literatura já estabelece a estreita relação entre o estado nutricional e o prognóstico dos pacientes ${ }^{1,2}$ .Segundo a Cystic Fibrosis Foundation (CFF), ${ }^{3}$ as crianças e os adolescentes com FC devem alcançar os mesmos parâmetros nutricionais daqueles saudáveis na mesma faixa etária. O acompanhamento nutricional, do crescimento e do desenvolvimento na população com FC, é fundamental para intervenção precoce e para o tratamento e reabilitação desses pacientes ${ }^{4,5}$.

O alcance e a manutenção de parâmetros nutricionais adequados na FC podem ser influenciados por diversos fatores. Balanço energético negativo devido à diminuição da ingesta calórica e da má absorção associada à insuficiência pancreática (IP); anorexia, infecções respiratórias recorrentes e inflamação acarretam aumento das demandas energéticas ${ }^{6,7 .}$ A desnutrição representa um importante fator de risco na progressão da doença e para a sobrevida nessa população ${ }^{4}$.

$O$ estado nutricional adequado de crianças e adolescentes com FC é, comumente, definido como o alcance de parâmetros antropométricos recomendados, obtidos através dos indicadores de Índice de Massa Corporal para idade (IMC/I) e Altura para Idade (A/I). Valores adequados destes parâmetros se associam com uma melhor função pulmonar e maior sobrevida desses pacientes ${ }^{8,10}$. Entretanto, estes indicadores apresentam limitações, pois refletem apenas o tamanho corporal e não distinguem seus principais componentes (tecido muscular e tecido adiposo) ${ }^{11 .}$

Atualmente, a avaliação da composição corporal pode ser realizada por diversos métodos, tais como: pesagem hidrostática, hidrometria, plestimografia, absortometria radiológica de dupla energia (DEXA) e antropometria. ${ }^{12}$ A antropometria é uma técnica de baixo custo, fácil execução e pouco invasiva. As medidas e os indicadores antropométricos de Circunferência do Braço (CB), Prega Cutânea Tricipital (PCT) e Circunferência Muscular do Braço (CMB) são bons preditores de composição corporal na FC e podem refletir, mais precocemente, mudanças no estado nutricional, durante o crescimento e/ou a reabilitação. ${ }^{13}$ Desta forma, este estudo objetivou avaliar o estado antropométrico de crianças e adolescentes com fibrose cística $(\mathrm{FC})$ e pesquisar sua associação com variáveis clínicas e demográficas.

\section{METODOLOGIA}

\section{Desenho e População do Estudo}

Trata-se de um estudo de corte transversal, incluindo-se todas as crianças e adolescentes com idades entre 1 -19 anos e diagnóstico confirmado de FC por dois testes do suor positivos (Cloro $\geq 60 \mathrm{mEq} / \mathrm{L}$ ) e/ou identificação de duas variantes patológicas no gene CFTR, acompanhados, por pelo menos três meses, em um centro de referência no tratamento da FC em um hospital universitário de Salvador. Os dados foram coletados durante as consultas de rotina, no período de julho de 2017 a abril de 2018. Foi realizada avaliação nutricional dos pacientes, de forma consecutiva, e preenchidos formulários com as informações clínicas e laboratoriais presentes nos prontuários médicos. Pacientes com morbidades associadas como diabetes mellitus, hipotireoidismo, síndrome de Down ou outras síndromes genéticas foram excluídos.

\section{Variáveis}

As seguintes variáveis foram estudadas: sexo, idade, idade do diagnóstico da FC, história de infecções respiratórias recorrentes, variantes identificadas no gene CFTR, níveis de elastase fecal, terapia de reposição enzimática (TRE) e avaliação nutricional. Para determinação da função pancreática, foi realizado o teste ELISA utilizando-se o kit Elastase Pancreárica 1 do fabricante ScheBO. A IP foi definida por valores de Elastase inferiores a $200 \mathrm{ug} / \mathrm{dL}$, conforme as orientações do fabricante para interpretação dos resultados, e/ou TRE.

A avaliação do estado nutricional foi realizada por um avaliador único treinado, sendo obtidos os indicadores $\mathrm{A} / \mathrm{l}$ e $\mathrm{IMC} / \mathrm{I}$, para todas as faixas etárias e o indicador $\mathrm{P} / \mathrm{I}$, apenas para os menores de 10 anos. Utilizou-se o critério escore $Z$ para estes indicadores, conforme as recomendações da Organização Mundial de Saúde ${ }^{14}$. Os cálculos dos escores Z foram feitos com os programas WHO Anthro para crianças menores de cinco anos e WHO Anthro PLUS para aquelas com cinco anos ou mais. As crianças menores de dois anos foram pesadas sem roupas em balança pediátrica digital da marca Filizola ${ }^{\circledR}$ (indústria S/A, São Paulo, Brasil) e as maiores de dois anos foram pesadas com o mínimo de roupas possível em uma balança de plataforma da marca Welmy ${ }^{\circledR}$ (indústria e comércio LTDA, SP, Brasil). A mensuração da estatura para os menores de 2 anos foi realizada deitando-se a criança no centro do infantômetro. Para pacientes com idade $\geq 2$ anos, a medição foi realizada com o indivíduo em pé e utilizando-se estadiômetro com escala em centímetros e precisão de um milímetro.

$\mathrm{Na}$ avaliação da composição corporal, a CB foi medida no ponto médio do braço direito relaxado, entre a projeção do acrômio e o olecrano, e a PCT foi aferida no ponto médio, na face posterior do braço direito, com a utilização do adipômetro clínico Cescorf ${ }^{\circledR}$ (Cescorf Equipamentos para Esporte Ltda - Porto Alegre, Rio Grande do Sul, Brasil), por três vezes, registrando-se o valor médio entre as três medidas. $O$ conteúdo de gordura foi avaliado pela PCT. Avaliou-se a massa muscular, calculando-se a CMB por meio da fórmula: $\mathrm{CMB}(\mathrm{cm})=\mathrm{CB}(\mathrm{cm})-(0,314 \times[(\mathrm{PCT}$ (mm) / 10]). Os resultados da CMB e da PCT foram comparados aos valores-padrão da National Center for Health Statistics (NCHS) demonstrados nas tabelas de percentis de Frisancho (1990), de acordo com sexo e idade, sendo considerados baixos os valores menores que o percentil 
5 , em risco entre os percentis 5 e 15, adequado entre os percentis 15 e 85 e em excesso acima do percentil $85^{15,16}$.

\section{Considerações éticas}

Este estudo faz parte de uma pesquisa maior denominada "Estudo clínico-epidemiológico da fibrose cística em um centro universitário de referência em Salvador, $B A^{\prime \prime}$ aprovado pelo Comitê de Ética em pesquisa, parecer no 121/2011. Foram obtidos termos de consentimento livre e esclarecido dos responsáveis ou dos pacientes, a depender da faixa etária e termos de assentimento, quando pertinente.

\section{Análise estatística}

Os dados obtidos foram registrados em formulário padrão e armazenados no programa Microsoft Excel ${ }^{\circledR}$ versão 365. Os dados descritivos foram expressos através de frequências simples e relativas das variáveis qualitativas estudadas, além das médias, desvio padrão, medianas e variações interquartílicas (IIQ), para variáveis quantitativas. Foram calculadas Razões de prevalências (RP) para avaliar associação entre a $\mathrm{CMB}<\mathrm{P} 5$ e as seguintes variáveis: sexo, idade do diagnóstico, variante F508del no gene CFTR, TRE e infecções respiratórias recorrentes.

\section{RESULTADOS}

Um total de 41 crianças e adolescentes foram estudadas, destes 22 (53,6\%) eram do sexo masculino. As medianas (Min-Max) das idades dos pacientes na admissão do estudo e no diagnóstico foram 78 meses (40-142) e 10 meses (4-68), respectivamente (Tabela 1 ).

Trinta e seis crianças $(87,8 \%)$ realizaram o teste de Elastase Fecal; destas, $23(63,9 \%)$ tiveram níveis de elastase $<200 \mu \mathrm{g} / \mathrm{g}$. Quatro crianças tinham queixa de esteatorreia e apresentavam desnutrição ou ganho de peso insuficiente ao diagnóstico; das quais, uma tinha nível normal de Elastase Fecal e as demais não realizaram o exame, assim um total de $27(65,8 \%)$ crianças faziam uso de TRE. Dezesseis (39,0\%) apresentavam infecções respiratórias recorrentes e todos os pacientes realizaram pesquisas de mutações no gene CFTR; destes, 38 (92,7\%) tiveram duas variantes patológicas identificadas, dos quais $25(65,8 \%)$ tinham pelo menos um alelo para a mutação F508del (Tabela 1).

De acordo com o IMC/I, a maioria dos indivíduos, $36(87,8 \%)$, foi classificada como eutrófica e apenas um $(2,4 \%)$ apresentava magreza por esse critério. A prevalência de crianças e adolescentes que apresentavam algum comprometimento da estatura e da massa muscular, avaliada pela $\mathrm{CMB}$, foi de $19,5 \%$ e $36,5 \%$, respectivamente.
Tabela 1 - Características demográficas, clínicas e laboratoriais de 41 crianças e adolescentes com fibrose cística estudados.

\begin{tabular}{|c|c|c|}
\hline Características & $\mathbf{N}(\%)$ & $\begin{array}{l}\text { Mediana (Min } \\
\text { - Max) }\end{array}$ \\
\hline \multicolumn{3}{|l|}{ Sexo } \\
\hline Feminino & $19(46,4 \%)$ & \\
\hline Masculino & $22(53,6 \%)$ & \\
\hline Idade (meses) & & $78(40-142)$ \\
\hline Idade ao diagnóstico ${ }^{1}$ (meses) & & $10(4-68)$ \\
\hline Crianças com diagnóstico $<12$ meses & $23(56,1 \%)$ & \\
\hline Crianças com diagnóstico $>12$ meses & $18(43,9 \%)$ & \\
\hline $\begin{array}{l}\text { História de Infecções respiratórias } \\
\text { recorrentes }\end{array}$ & $16(39,0 \%)$ & \\
\hline \multicolumn{3}{|l|}{ Níveis de Elastase ${ }^{2}(\mu \mathrm{g} / \mathrm{g})$} \\
\hline$<200$ & $23(63,9 \%)$ & \\
\hline$>200$ & $13(36,1 \%)$ & \\
\hline TRE & $27(65,8 \%)$ & \\
\hline \multicolumn{3}{|l|}{ Variante F508del } \\
\hline Homozigotos & $11(28,9 \%)$ & \\
\hline Heterozigotos & $14(36,9 \%)$ & \\
\hline
\end{tabular}

TRE=Terapia de Reposição enzimática; os dados expressos nesta tabela representam os valores totais e porcentagens (\%). 1. Quatro crianças tiveram diagnóstico pela Triagem Neonatal e duas por história familiar da doença. 2. Cinco pacientes não realizaram teste de Elastase.

Fonte: dados da pesquisa

Através da aferição da PCT, 28 (68,2\%) indivíduos apresentavam reserva de tecido adiposo adequada e seis $(14,1 \%)$ tinham valores acima média. Do total de crianças e adolescentes que tinham IP, 12 (44,4\%) eram desnutridos pelo critério da CMB e nenhuma pelo IMC/I. Dos 36 $(87,8 \%)$ pacientes considerados eutróficos pelo IMC/I, 13 $(36,1 \%)$ tinham déficit de massa muscular avaliada pela CMB e $7(19,4 \%)$ comprometimento da estatura pelo critério da A/I (Tabela 2).

Tabela 2 - Classificação do estado Nutricional de 41 crianças e adolescentes com fibrose cística estudados.

\begin{tabular}{|c|c|}
\hline Classificação & N (\%) \\
\hline $\mathrm{P} / \mathrm{I}^{1}$ Z-escore - Baixo Peso & $2(7,4 \%)$ \\
\hline $\mathrm{P} / \mathrm{I}^{1} \mathrm{Z}$ - escore - Peso Adequado & $25(92,6 \%)$ \\
\hline A/I, Z-escore - Muito baixa estatura & $2(4,9 \%)$ \\
\hline A/I, Z-escore - Baixa estatura & $6(14,6 \%)$ \\
\hline A/I, Z-escore - Estatura Adequada & $33(80,5 \%)$ \\
\hline IMC/I, Z-escore- Magreza & $1(2,4 \%)$ \\
\hline IMC/I, Z-escore - Eutrofia & $36(87,8 \%)$ \\
\hline IMC/I, Z-escore - Sobrepeso & $4(9,7 \%)$ \\
\hline CMB, percentil $<5$ & $15(36,6 \%)$ \\
\hline $\mathrm{CMB}$, percentil 15-85 & $18(43,9 \%)$ \\
\hline $\mathrm{CMB}$, percentil > 85 & $8(19,5 \%)$ \\
\hline $\mathrm{PCT}$, percentil $<5$ & $4(9,7 \%)$ \\
\hline PCT, percentil 10-15 & $3(7,3 \%)$ \\
\hline PCT, percentil $15-85$ & $28(68,2 \%)$ \\
\hline PCT, percentil > 85 & $6(14,1 \%)$ \\
\hline
\end{tabular}

$N=$ número total de participantes; $P / I=P e s o / I d a d e ; A / I=A / t u r a / l d a d e ;$ $I M C / I=I M C / I d a d e ; C M B=$ Circunferência muscular do Braço; $P C T=$ Prega cutânea tricipital.1.P/I = 27 pacientes tinham idade inferior $a$ dez anos.

Fonte: dados da pesquisa 
Entre os 16 (39,0\%) pacientes que apresentavam infecções respiratórias recorrentes, quatro $(25,0 \%)$ apresentavam $\mathrm{CMB}<\mathrm{P} 5$, um $(5,8 \%)$ tinha IMC/I $<-2$ (magreza) e três $(17,7 \%)$ tinham algum comprometimento de estatura. A Tabela 3 apresenta a classificação nutricional dos pacientes de acordo com as variáveis clínicas de infecções respiratórias recorrentes e uso de TRE.

Através do cálculo de razão de prevalência (RP), observou-se associação entre CMB <P5 com: reserva de tecido adiposo adequada ou acima da média (PCT >P15), comprometimento da estatura, TRE e a mutação F508del, sendo também mais comum no sexo masculino e em crianças que tiveram o diagnóstico antes dos 12 meses. $O$ comprometimento do estado nutricional, pela análise da composição corporal, esteve mais fortemente associado com o uso da TRE (Tabela 4).

Tabela 3 - Classificação dos parâmetros antropométricos do estado nutricional de acordo com o uso de Terapia de reposição enzimática (TRE) e a história de infecções respiratórias recorrentes dos pacientes com fibrose cística estudados ( $N=41$ ).

\begin{tabular}{|c|c|c|}
\hline \multirow{3}{*}{$\begin{array}{l}\text { Medidas } \\
\text { do Estado } \\
\text { Nutricional }\end{array}$} & \multicolumn{2}{|c|}{ Variáveis Clínicas } \\
\hline & $\begin{array}{l}\text { TRE }^{2} \\
\mathrm{~N}(\%)\end{array}$ & $\begin{array}{c}\text { Infecções Respiratórias } \\
\text { Recorrentes } \\
\text { N(\%) } \\
\end{array}$ \\
\hline & $(n=27)$ & $(n=16)$ \\
\hline \multicolumn{3}{|l|}{ Peso//dade ${ }^{1}$} \\
\hline Baixo Peso & $2(7,4 \%)$ & $1(6,2 \%)$ \\
\hline Peso adequado & $18(66,6 \%)$ & $7(43,7 \%)$ \\
\hline \multicolumn{3}{|l|}{ Altura/Idade } \\
\hline Muito baixa estatura & $2(7,4 \%)$ & $1(6,2 \%)$ \\
\hline Baixa estatura & $4(14,9 \%)$ & $2(12,5 \%)$ \\
\hline Estatura adequada & $21(77,8 \%)$ & $13(81,2 \%)$ \\
\hline \multicolumn{3}{|l|}{ IMC/Idade } \\
\hline Magreza & $0(0,0 \%)$ & $1(6,2 \%)$ \\
\hline Eutrofia & $24(88,9 \%)$ & $14(87,5 \%)$ \\
\hline Sobrepeso & $3(11,1 \%)$ & $1(6,2 \%)$ \\
\hline \multicolumn{3}{|l|}{ РCT } \\
\hline$<\mathrm{P} 5$ & $4(14,8 \%)$ & $1(6,2 \%)$ \\
\hline P5-P15 & $2(7,4 \%)$ & $2(12,5 \%)$ \\
\hline P15-P85 & $19(70,3 \%)$ & $10(62,5 \%)$ \\
\hline > P85 & $2(7,4 \%)$ & $3(18,8 \%)$ \\
\hline \multicolumn{3}{|l|}{ CMB } \\
\hline$<\mathrm{P} 5$ & $12(44,4 \%)$ & $4(25,0 \%)$ \\
\hline P15-P85 & $12(44,4 \%)$ & $8(50,0 \%)$ \\
\hline > P85 & $3(11,2 \%)$ & $4(25,0 \%)$ \\
\hline
\end{tabular}

$N=$ número total de participantes; $P / I=P e s o / I d a d e ; A / I=A l t u r a / I d a d e ;$ $I M C / I=I M C / I d a d e ; P C T=$ prega cutânea tricipital; $C M B=$ Circunferência Muscular do Braço; os dados expressos nesta tabela representam os valores totais e porcentagens (\%).

1. Crianças de 0 a 10 anos $N=27$ pacientes

2. Número de pacientes que faziam uso de TRE: 27

3. Número de pacientes que apresentavam infecções respiratórias recorrentes: 16

Fonte: Dados da pesquisa

Rev. Ciênc. Méd. Biol., Salvador, v. 19, n. 2, p. 298-304, mai./ago. 2020
Tabela 4 - Avaliação da associação entre $C M B<P 5^{1}$ e variáveis clínicas de 41 crianças e adolescentes com Fibrose Cística.

\begin{tabular}{|c|c|c|c|c|}
\hline & $\mathbf{N}$ & $\begin{array}{l}\text { CMB } \\
<\mathrm{P} 5\end{array}$ & Prevalência & $\begin{array}{l}\text { Razão de } \\
\text { Prevalência }\end{array}$ \\
\hline \multicolumn{5}{|l|}{ Sexo } \\
\hline Masculino & 22 & 9 & $40,9 \%$ & 1,29 \\
\hline Feminino & 19 & 6 & $31,6 \%$ & \\
\hline \multicolumn{5}{|c|}{ Idade do diagnóstico } \\
\hline$<12$ meses $^{2}$ & 17 & 7 & $41,2 \%$ & 1,23 \\
\hline$>12$ meses & 18 & 6 & $33,3 \%$ & \\
\hline \multicolumn{5}{|l|}{ TRE } \\
\hline Sim & 27 & 12 & $44,4 \%$ & 6,25 \\
\hline Não & 14 & 1 & $7,1 \%$ & \\
\hline \multicolumn{5}{|c|}{$\begin{array}{l}\text { Infecções respiratórias } \\
\text { recorrentes }\end{array}$} \\
\hline Sim & 16 & 4 & $25,0 \%$ & 0,56 \\
\hline Não & 25 & 11 & $44,0 \%$ & \\
\hline \multicolumn{5}{|l|}{$A / 1<-2$} \\
\hline Sim & 8 & 5 & $62,5 \%$ & 2,06 \\
\hline Não & 33 & 10 & $30,3 \%$ & \\
\hline \multicolumn{5}{|l|}{ PCT > P15 } \\
\hline $\operatorname{Sim}$ & 34 & 13 & $38,2 \%$ & 1,33 \\
\hline Não & 7 & 2 & $28,6 \%$ & \\
\hline \multicolumn{5}{|c|}{ Mutação F508/del ${ }^{3}$} \\
\hline Sim & 25 & 12 & $48,0 \%$ & 3,12 \\
\hline Não & 13 & 2 & $15,4 \%$ & \\
\hline
\end{tabular}

$N=$ número total de participantes; $A / I=A /$ tura/Idade; $P C T=$ prega cutânea tricipital; $C M B=$ Circunferência Muscular do Braço; os dados expressos nesta tabela representam os valores totais e porcentagens (\%).

1. $N=15$ pacientes com $C M B<P 5 ; 2.6$ pacientes foram excluídos dessa associação por terem o diagnóstico direcionado pela triagem neonatal ou pela história familiar da doença. 3. Um total de 38 pacientes tiveram mutações identificadas, destes $25(65,8 \%)$ tinham pelo menos um alelo para mutação F508/del.

Fonte: Dados da pesquisa

\section{DISCUSSÃO}

Os fatores que determinam fenótipos mais graves da FC, com apresentações clinicas variáveis, acarretam em dificuldades no ganho de peso, no crescimento linear e na manutenção de um estado nutricional adequado na infância ${ }^{1}$. A desnutrição é uma achado frequente nesses pacientes, tornando-se ainda mais prevalente em adultos, e é um indicador de pior prognóstico da doença ${ }^{2,8,16}$.

No presente estudo observou-se maior prevalência de desnutrição adotando-se como critério a $\mathrm{CMB}$ do que o IMC/I, apesar de o último ser mais comumente utilizado para diagnóstico nutricional dos pacientes com $\mathrm{FC}$, nessa faixa etária ${ }^{1,4,6,17}$.Este achado é semelhante ao encontrado em um estudo realizado na região Sudeste do país. ${ }^{18}$

A análise antropométrica da composição corporal, por meio de pregas cutâneas e pelo cálculo da CMB, é 
um método factível, de baixo custo e fácil aplicação. Os resultados deste estudo concordam com dados prévios que demonstram maior sensibilidade no diagnóstico da desnutrição pela análise da composição corporal, por métodos como Bioimpedância Elétrica (BIA) e avaliação antropométrica de pregas cutâneas e das circunferências comparadas ao uso isolado do indicador $\mathrm{IMC} / \mathrm{I}^{12,18-21}$.

As diretrizes nacionais e internacionais recomendam avaliação de pregas cutâneas, circunferência do braço e bioimpedância como métodos adicionais para determinação do estado nutricional ${ }^{3,4,22}$. Em vista que o peso corporal é dividido em massa gorda e massa livre de gordura (reserva proteica), a avaliação desses dois compartimentos pode identificar precocemente mudanças no estado nutricional que não podem ser avaliadas pelos indicadores obtidos através das medidas de peso e altura isoladamente $\mathrm{e}^{11,23}$.

Os fatores inerentes à fisiopatologia da doença, bem como suas manifestações clínicas, por si só, exercem um forte efeito na sobrevida dos pacientes. Evidências suportam redução do risco de mortalidade em pacientes que têm melhores parâmetros de crescimento e de desenvolvimento, tanto pelo ganho de peso adequado e, particularmente, pelo bom crescimento linear ${ }^{1,8}$. A baixa estatura, por representar uma desnutrição crônica, é apontada como um importante e independente fator de risco para mortalidade na $\mathrm{FC}$, por influenciar diretamente no crescimento e no desenvolvimento pulmonar ${ }^{24}$.

Neste estudo, a baixa estatura $(\mathrm{A} / \mathrm{I}<-2)$ ocorreu em oito $(19,5 \%)$ crianças; das quais, seis $(75 \%)$ faziam TRE, corroborando o maior risco de comprometimento do estado nutricional para os pacientes com IP. Entre as crianças com baixa estatura, sete $(87,5 \%)$ foram classificadas como eutróficas pelo IMC/I, mesmo tendo comprometimento crônico do estado nutricional. Estes achados enfatizam a importância da utilização de outros indicadores na avaliação nutricional desses pacientes.

Nesse estudo foi encontrada uma associação entre comprometimento da estatura e $\mathrm{CMB}<\mathrm{P} 5$, corroborando a hipótese de que existe aumento do catabolismo proteico em crianças e adolescentes que apresentam um crescimento linear deficiente ${ }^{24}$ Estudos prévios apontam que valores adequados de $\mathrm{A} / \mathrm{I}$ associam-se ao menor número de hospitalizações e ao retardo do início dos sintomas respiratórios na $\mathrm{FC}^{24,25}$.

$\mathrm{O}$ crescimento pode ser influenciado por diversos fatores não avaliados neste estudo como os genéticos, distúrbios endócrinos, ingestão alimentar, número de internamentos e função pulmonar. Além disso, a velocidade de crescimento nas diferentes faixas de idade não foi avaliada. No entanto, os achados enfatizam a importância da utilização do indicador A/I na avaliação nutricional desses pacientes visto a alta prevalência de baixa estatura.

A depleção do estado nutricional na FC é resultado da associação de diversos fatores ligados ao fenótipo da doença, tais como: a presença de insuficiência pancreática com má absorção crônica, infecções respiratórias recorrentes, aumento das demandas e do gasto energético e baixa ingestão alimentar, além da baixa adesão ao tratamento e a presença de comorbidades ${ }^{2,6}$. $O$ ganho de peso e a manutenção da massa muscular dentro da normalidade estão ligados a um crescimento adequado, melhor função pulmonar e maior sobrevida dos pacientes $^{25}$. A perda de massa muscular, comum na FC, estimada de forma indireta pela análise da $\mathrm{CMB}$, pode afetar a força e a resistência dos músculos respiratórios, bem como comprometer a função imunitária $2,8,25$.

A literatura descreve a depleção preferencial de massa muscular e a preservação do tecido adiposo na FC, achados que se associam com o aumento das exacerbações pulmonares ${ }^{11,23}$. De forma concordante, o presente estudo demonstrou que a maioria da população $(82,9 \%)$ avaliada apresentava uma reserva de tecido adiposo adequada ou acima da média. Destaque-se que as intervenções nutricionais na $\mathrm{FC}$ são direcionadas ao aumento de peso corporal e manutenção de IMC/I adequado, com dietas de alta densidade energética, principalmente com alto percentual de gordura ${ }^{25}$. Já é levantada a hipótese que o suporte nutricional necessário na FC preserva a massa adiposa ao contrário da massa muscular, que sofre maiores impactos do estado catabólico crônico da doença $a^{1,2,23}$.

Muitos estudos já destacaram a influência do estado nutricional na evolução da doença, no comprometimento da função pulmonar e na maior frequência de infecções respiratórias entre os pacientes com $\mathrm{FC}^{8-10,17}$. O número e intensidade das exacerbações pulmonares desses pacientes determinam o estado crônico inflamatório e de estresse, acarretando em aumento das demandas energéticas e da proteólise muscular ${ }^{23,26}$. Entretanto este estudo não encontrou uma associação quanto a presença de infecções respiratórias recorrentes e a desnutrição pela CMB. Esse achado pode decorrer do pequeno número de pacientes avaliados, da faixa etária estudada e pela relativa precocidade do diagnóstico da doença na população estudada.

Outro fator prognóstico importante para a desnutrição na FC é a insuficiência pancreática (IP), presente, em média, em $85 \%$ dos $\operatorname{casos}^{27,28}$. Este estudo encontrou uma prevalência de IP em $65,8 \%$ da população que se associou fortemente com a desnutrição pela avaliação da $\mathrm{CMB}(\mathrm{P}<5)$, destacando a importância da prevenção ou da identificação precoce do comprometimento nutricional nessa população. ${ }^{28}$

Mutações mais graves se associam a um fenótipo de IP, pior função pulmonar, maior comprometimento do estado nutricional, menor sobrevida dos pacientes e a um pior prognóstico ${ }^{29,30}$. A variante F508del (mutação de classe II) é a mais frequente mundialmente, ocorrendo em cerca de $70 \%$ dos alelos, associando-se a fenótipos mais graves ${ }^{3}$. Esse estudo identificou esta variante em cerca de $65,8 \%$ dos alelos estudados e uma associação desse achado com $\mathrm{CMB}<\mathrm{P5}$, o que concorda com estudo anteriormente realizado ${ }^{30}$. 
Esse estudo apresenta diversas limitações, dentre as quais podemos destacar: o pequeno número de pacientes avaliados em um único centro, a ampla faixa etária estudada, a coleta dos dados em um único momento, a ausência de registros do consumo alimentar e o desenho do estudo, não permitindo estabelecer relações causais entre as variáveis e os desfechos clínicos. Entretanto, foi possível demonstrar que o indicador antropométrico CMB foi mais sensível na identificação da desnutrição e do risco nutricional na população estudada, bem como sua associação com variáveis demográficas e clínicas, o que pode contribuir na identificação dos pacientes com maior risco nutricional e na intervenção mais precoce.

\section{CONCLUSÕES}

$\mathrm{O}$ indicador IMC/I pode subestimar a prevalência de desnutrição. Além disso, por não avaliar separadamente os compartimentos corporais, este indicador pode ser resultado de uma adequada ou excessiva reserva de tecido adiposo, não detectando depleção de massa livre de gordura. Desta forma, conclui-se que o IMC pode não ser suficiente para a avaliação do estado nutricional na FC e que a avaliação da composição corporal através do indicador $\mathrm{CMB}$ pode ser facilmente incorporada na rotina de acompanhamento das crianças com FC.

\section{REFERÊNCIAS}

1. LUSMAN, S.; SULLIVAN, J. Nutrition and growth in cystic fibrosis. Pediatr. Clin., Philadelphia, v. 63, n. 4, p. 661-678, 2016.

2. MATEL, J.L.; MILLA, C.E. Nutrition in cystic fibrosis. In: Seminars in respiratory and critical care medicine. 2009. p. 579-586.

3. CYSTIC FIBROSIS FOUNDATION. Cystic Fibrosis Foundation Patient Registry Annual Data Report 2017. Disponível em: http://www.cff.org/. Acesso em: 20 Sept. 2018.

4. STALLINGS, V.A. et al. Evidence-based practice recommendations for nutrition-related management of children and adults with cystic fibrosis and pancreatic insufficiency: results of a systematic review. J. Am. Diet. Assoc., Chicago, v. 108, n. 5, p. 832-839, 2008.

5. ISA, H.M.; AL-ALI, L.F.; MOHAMED, A.M. Growth assessment and risk factors of malnutrition in children with cystic fibrosis. Saudi med. j., Ryadh, v. 37, n. 3, p. 293-298, 2016.

6. GASKIN, K. J. Nutritional care in children with cystic fibrosis: are our patients becoming better?. Eur j. clin. nutr., London, v. 67, n. 5, p. 558-564, 2013.

7. ROSA, F.R. et al. Fibrose cística: uma abordagem clínica e nutricional. Rev. Nutr., Campinas, v. 21, n. 6, p. 725-737, 2008.

8. HAUSCHILD, D. B. et al. Associação do estado nutricional com função pulmonar e morbidade em crianças e adolescentes com fibrose cística: coorte de 36 meses. Rev. Paul. Pediatr. São Paulo, v. 36, n. 1, p. 31-38, 2018

9. BARJA, S. et al. Evolución nutricional y función pulmonar en niños y adolescentes chilenos con fibrosis quística. Rev .méd. Chile, Chile, v. 139, n. 8, p. 977-984, 2011.

10. STEINKAMP, G.; WIEDEMANN, B. Relationship between nutritional status and lung function in cystic fibrosis: cross sectional and longitudinal analyses from the German CF quality assurance (CFQA) project. Thorax, London, v. 57, n. 7, p. 596-601, 2002.

11. ALVAREZ, J.A. et al. Body composition and lung function in cystic fibrosis and their association with adiposity and normal-weight obesity. Nutrition, [s.l], v. 32, n. 4, p. 447-452, 2016.

12. SANT'ANNA, M.S.L.; PRIORE, S.E.; FRANCESCHINI, S.C.C. Métodos de avaliação da composição corporal em crianças. Rev. Paul. Pediatr., São Paulo, v. 27, n. 3, p. 315-321, 2009.

13. MAUCH, R.M. et al. Associação dos parâmetros de crescimento e nutricionais com função pulmonar na fibrose cística: revisão da literatura. Rev. Paul. Pediatr., São Paulo, v. 34, n. 4, p. 503-509, 2016.

14. WORLD HEALTH ORGANIZATION. WHO child growth standards: length/height-for-age, weight-for-age, weight-for-length, weight-forheight and body mass index-for-age: methods and development. 2006.

15. FRISANCHO, A. R. Anthropometric standards for the assessment of growth and nutritional status. University of Michigan press, 1990.

16. CENTER FOR DISEASE CONTROL AND PREVENTION (CDC). National Center For Health Statistics NCHS. Atlanta: 2000-2004. growth charts. Disponível em: http://www.cdc.gov/growthcharts. Acesso em: 10 ago. 2018.

17. MACHOGU, E. et al. Comparison of WHO and CDC growth charts in predicting pulmonary outcomes in cystic fibrosis. J. pediatr. gastroenterol. nutr., New York, v. 60, n. 3, p. 378, 2015.

18. CHAVES, C.R.M.M. et al. Associação entre medidas do estado nutricional e a função pulmonar de crianças e adolescentes com fibrose cística. J. bras. pneumol., Brasília,v. 35, n. 5, p. 409-414, 2009.

19. GROENEWEG, M. et al. Assessment of nutritional status in children with cystic fibrosis: conventional anthropometry and bioelectrical impedance analysis. A cross-sectional study in Dutch patients. J Cystic Fibrosis, [s.I], v. 1, n. 4, p. 276-280, 2002.

20. DOULGERAKI, A. et al. Body composition and lung function in children with cystic fibrosis and meconium ileus. Eur. J. Pedriatr., Heldeberg, v. 176, n. 6, p. $737-743,2017$.

21. ALICANDRO, G. et al. Estimating body composition from skinfold thicknesses and bioelectrical impedance analysis in cystic fibrosis patients. J Cyst. Fibros., Amsterdam, v. 14, n. 6, p. 784-791, 2015.

22. ATHANAZIO, R.A. et al. Diretrizes brasileiras de diagnóstico e tratamento da fibrose cística. J. bras. pneumol., Brasilia, v. 43, n. 3, p. 219-245, 2017

23. KING, S.J. et al. Fat-free mass depletion in cystic fibrosis: associated with lung disease severity but poorly detected by body mass index. Nutrition, v. 26, n. 7-8, p. 753-759, 2010.

24. BEKER, L.T.; RUSSEK-COHEN, E.; FINK, R.J. Stature as a prognostic factor in cystic fibrosis survival. J Am. Diet. Assoc., Chicago, v. 101, n. 4, p. 438-442, 2001.

25. UMŁAWSKA, W. et al. Effect of selected factors associated with the clinical corse of disease on nutritional status in children with cystic fibrosis. Adv. Clin. Exp.I Med., Wroclam, v. 23, n. 5, p. 775-783, 2014.

26. SOUZA, R. P. et al. Uso da ultrassonografia para avaliar a espessura muscular e a gordura subcutânea em crianças e adolescentes com fibrose cística. Rev. Paul. Pediatr., São Paulo, v. 36, n. 4, p. 457-465, 2018.

27. NERI, L.C.L. et al. Avaliação do perfil nutricional em pacientes portadores de fibrose cística de acordo com faixa etária. Rev. Paul. Pediatr., São Paulo, v. 37, n. 1, p. 58-64, 2019.

28. CHAVES, C.R.M.M. et al. Estado nutricional e distribuição de gordura 
corporal em crianças e adolescentes com Fibrose Cística. Ciênc. Saúde Colet., Rio de Janeiro, v. 20, p. 3319-3328, 2015.

29. TRIDELLO, G. et al. Early diagnosis from newborn screening maximises survival in severe cystic fibrosis. ERJ Open Res., [s.I], v. 4, n. 2, 2018.
30. SZWED, A. et al. Survival of patients with cystic fibrosis depending on mutation type and nutritional status. In: Pulmonary Disorders and Therapy. Springer: Cham, 2017. p. 65-72.

Submetido em: $27 / 04 / 2020$

Aceito em: 25/06/2020 